\title{
On the Global Convergence of the PERRY-SHANNO Method for Nonconvex Unconstrained Optimization Problems*
}

\author{
Linghua Huang ${ }^{1}$, Qingjun $\mathrm{Wu}^{2}$, Gonglin Yuan ${ }^{3}$ \\ ${ }^{1}$ Department of Mathematics and Statistics, Guangxi University of Finance and Economics, Nanning, China \\ ${ }^{2}$ Department of Mathematics and Computer Science, Yulin Normal University, Yulin, China \\ ${ }^{3}$ Department of Mathematics and Information Science, Guangxi University, Nanning, China \\ E-mail: linghuahuang@163.com,wqj600@yahoo.com.cn, glyuan@gxu.edu.cn \\ Received November 15, 2010; revised January 12, 2011; accepted January 16, 2011
}

\begin{abstract}
In this paper, we prove the global convergence of the Perry-Shanno’s memoryless quasi-Newton (PSMQN) method with a new inexact line search when applied to nonconvex unconstrained minimization problems. Preliminary numerical results show that the PSMQN with the particularly line search conditions are very promising.
\end{abstract}

Keywords: Unconstrained Optimization, Nonconvex Optimization, Global Convergence

\section{Introduction}

We consider the unconstrained optimization problem:

$$
\min \left\{f(x) \mid x \in R^{n}\right\},
$$

where $f: R^{n} \rightarrow R$ is continuously differentiable. Perry and Shanno's memoryless quasi-Newton method is often used to solve the problem (1.1) when $n$ is large. The PSMQN method was originated from the works of Perry (1977 [1]) and Shanno (1978 [2]), and subsequently developed and analyzed by many authors. Perry and Shanno's memoryless method is an iterative algorithm of the form

$$
x_{k+1}=x_{k}+\alpha_{k} d_{k} \text {, }
$$

where $\alpha_{k}$ is a steplength, and $d_{k}$ is a search direction which given by the following formula:

$$
\begin{aligned}
& d_{1}=-g_{1}, \\
& d_{k+1}=-\frac{y_{k}^{T} s_{k}}{\left\|y_{k}\right\|^{2}} g_{k+1}+\left(\frac{y_{k}^{T} g_{k+1}}{\left\|y_{k}\right\|^{2}}-2 \frac{s_{k}^{T} g_{k+1}}{y_{k}^{T} s_{k}}\right) s_{k} \\
&+\frac{s_{k}^{T} g_{k+1}}{\left\|y_{k}\right\|^{2}} y_{k}, k \geq 1
\end{aligned}
$$

where $s_{k}=x_{k+1}-x_{k}, y_{k}=g_{k+1}-g_{k}$ and $g_{j}$ denotes the

*Foundation Item: This work is supported by Program for Excellent Talents in Guangxi Higher Education Institutions, China NSF grands 10761001, Guangxi Education research project grands 201012MS013, and Guangxi SF grands 0991028. gradient of $f$ at $x_{j}$. If we denote

$$
B_{k+1}=\frac{\left\|y_{k}\right\|^{2}}{y_{k}^{T} s_{k}} I+\frac{y_{k} y_{k}^{T}}{y_{k}^{T} s_{k}}-\frac{\left\|y_{k}\right\|^{2}}{s_{k}^{T} y_{k}\left\|S_{k}\right\|^{2}} S_{k} s_{k}^{T}
$$

and

then

$$
H_{k+1}=B_{k+1}^{-1},
$$

$$
H_{k+1}=\frac{y_{k}^{T} s_{k}}{\left\|y_{k}\right\|^{2}} I+2 \frac{s_{k} s_{k}^{T}}{y_{k}^{T} s_{k}}-\frac{1}{\left\|y_{k}\right\|^{2}}\left(s_{k} y_{k}^{T}+y_{k} s_{k}^{T}\right) .
$$

By (1.4) and (1.5), we can rewrite $d_{k+1}$ as

$$
d_{k+1}=-H_{k+1} g_{k+1} \text {. }
$$

In practical testing, it is shown that the memoryless method is much more superior to the conjugate gradient methods, and in theoretic analysis, Perry and Shanno had proved that this method will be convergent for uniform convex function with Armijor or Wolfe line search. Shanno pointed out that this method will be convergent for convex function if the Wolfe line search is used. Despite of many efforts has been put to its convergence behavior, the global convergence of the PSMQN method is still open for the case of $\mathrm{f}$ is not a convex function. Recently, Han, Liu and Yin [3] proved the global convergence of the PSMQN method for nonconvex function under the following condition

$$
\lim _{k \rightarrow \infty}\left\|s_{k}\right\|=0 .
$$


The purpose of this paper is to study the global convergence behavior of PSMQN method by introducing a new line search conditions. The line search strategy used in this paper is as follows: find $t_{k}$ satisfying

$$
f\left(x_{k}+t_{k} d_{k}\right)-f\left(x_{k}\right) \leq \delta t_{k} g_{k}^{T} d_{k}-\min \left\{\varepsilon_{1},\left\|g_{k}\right\|^{\mu}\right\} t_{k}^{2}\left\|d_{k}\right\|^{4}
$$

and

$$
g\left(x_{k}+t_{k} d_{k}\right)^{T} d_{k} \geq \sigma g_{k}^{T} d_{k},
$$

where $\varepsilon_{1} \in(0,1)$ s a small scalar and $\mu \in(1,+\infty)$ is a large scalar. It is clear that (1.7) and (1.8) are a modification of the weak Wolfe-Powell (MWWP) line search conditions.

This paper is organized as follows. In Section 2, we present the PSMQN with the new line search MWWP. In Section 3, we establish the global convergence of the proposed method. The preliminary numerical results are contained in Section 4.

\section{Algorithm}

By combining the PSMQN and the MWWP, we can obtain a modified Perry-Shanno's memoryless quasi-Newton method as follows:

Algorithm 1 (A Modified PSMQN method: MPSMQN)

Step 0: Given $x_{1} \in R^{n}$, set $d_{1}=-g_{1}, k=1$. If $g_{1}=0$, then stop.

Step 1: Find a $t_{k}>0$ satisfying MWWP.

Step 2: Let $x_{k+1}=x_{k}+t_{k} d_{k}$ and $g_{k+1}=g\left(x_{k+1}\right)$. If $g_{k+1}=0$, then stop.

Step 3: Generate $d_{k+1}$ by the PSMQN formula (1.4).

Step 4: Set $k:=k+1$, go to Step 1 .

\section{Global Convergence}

In order to prove the global convergence of Algorithm 1, we will impose the following two assumptions, which have been used often in the literature to analyze the global convergence of conjugate gradient and quasi-Newton methods with inexact line searches.

Assumption A. The level set

$$
\Omega=\left\{x \in R^{n} \mid f(x) \leq f\left(x_{1}\right)\right\}
$$

is bounded.

Assumption B. There exists a constant $L$ such that for any $x, y \in \Omega$,

$$
\|g(x)-g(y)\| \leq L\|x-y\| .
$$

Since $\left\{f\left(x_{k}\right)\right\}$ is a decreasing sequence, it is clear that the sequence $\left\{x_{k}\right\}$ generated by Algorithm 1 is contained in, and there exists a constant $f^{*}$, such that

$$
\lim _{k \rightarrow \infty} f\left(x_{k}\right)=f^{*} .
$$

Lemma 3.1 Suppose that Assumption A holds and there exists a positive constant $\varepsilon$ such that

$$
\left\|g_{k}\right\| \geq \varepsilon \text {. }
$$

then,

$$
\lim _{k \rightarrow \infty} t_{k}\left\|d_{k}\right\|^{2}=0
$$

and

$$
\lim _{k \rightarrow \infty} t_{k}\left\|d_{k}\right\|^{2}=0 .
$$

Proof. From (3.2), we have

$$
\begin{aligned}
\sum_{k=1}^{\infty}\left(f\left(x_{k}\right)-f\left(x_{k+1}\right)\right) & =\lim _{N \rightarrow \infty} \sum_{k=1}^{N}\left(f\left(x_{k}\right)-f\left(x_{k+1}\right)\right) \\
& =\lim _{N \rightarrow \infty}\left(f\left(x_{1}\right)-f\left(x_{k+1}\right)\right) \\
& =f\left(x_{1}\right)-f^{*} .
\end{aligned}
$$

thus

$$
\sum_{k=1}^{\infty}\left(f\left(x_{k}\right)-f\left(x_{k+1}\right)\right)<+\infty,
$$

which combining with

$$
f\left(x_{k}+t_{k} d_{k}\right)-f\left(x_{k}\right) \leq \delta t_{k} g_{k}^{T} d_{k}-\min \left\{\varepsilon_{1},\left\|g_{k}\right\|^{\mu}\right\} t_{k}^{2}\left\|d_{k}\right\|^{4}
$$

and (3.3), yields

$$
\sum_{k=1}^{\infty} t_{k}^{2}\left\|d_{k}\right\|^{4}<+\infty
$$

and

$$
\sum_{k=1}^{\infty}-t_{k} g_{k}^{T} d_{k}<+\infty
$$

therefore, (3.4) and (3.5) hold.

The property (3.4) is very important for proving the global convergence of Algorithm 1, and it is not known yet for us whether (3.4) holds for other types line search (for example, the weak Wolfe-Powell conditions or the strong Wolfe-Powell conditions).

By using (3.4) and the result given in [3], we can deduce that Algorithm 1 is convergent globally. In the following, we give a direct proof for the global convergence of Algorithm 1.

Lemma 3.2 Assume that $B_{k}$ is a positive definite matrix. Then

$$
\frac{\left\|g_{k}\right\|^{2}}{g_{k}^{T} H_{k} g_{k}} \geq T_{r}\left(B_{k}\right) \text {. }
$$

Proof. Omitted.

Lemma 3.3 Supposed that Assumption A and B hold, and $x_{k}$ is generated by Algorithm 1.Then there exists a positive constant $c$ such that 


$$
\sum_{i=1}^{k} t_{k} \geq c k
$$

Proof. By (1.5) and (1.6), we have

$$
T_{r}\left(B_{k+1}\right)=n \frac{\left\|y_{k}\right\|^{2}}{y_{k}^{T} s_{k}},
$$

and

$$
T_{r}\left(H_{k+1}\right)=(n-2) \frac{\left\|y_{k}\right\|^{T} s_{k}}{\left\|y_{k}\right\|^{2}}+2 \frac{\left\|S_{k}\right\|^{2}}{y_{k}^{T} s_{k}} .
$$

From $\left(y_{k}^{T} s_{k}\right)^{2} \leq\left\|s_{k}\right\|^{2}\left\|y_{k}\right\|^{2}$, we obtain

$$
T_{r}\left(H_{k+1}\right)=(n-2) \frac{\left\|y_{k}\right\|^{T} s_{k}}{\left\|y_{k}\right\|^{2}}+2 \frac{\left\|S_{k}\right\|^{2}}{y_{k}^{T} s_{k}} .
$$

Using (1.8) and (3.12), we have

$$
T_{r}\left(H_{k+1}\right) \leq \frac{n}{1-\beta} \frac{t_{k}\left\|H_{k} g_{k}\right\|^{2}}{g_{k}^{T} H_{k} g_{k}} .
$$

By using the positive definiteness of $H_{k}$, we have

$$
\frac{\left\|H_{k} g_{k}\right\|^{2}}{g_{k}^{T} H_{k} g_{k}} \leq T_{r}\left(H_{k}\right),
$$

from which we can deduce that

$$
T_{r}\left(H_{k+1}\right) \leq\left(\frac{n}{1-\beta}\right)^{k} T_{r}\left(H_{1}\right) \prod_{i=1}^{k} t_{k} .
$$

By the Assumption B, (3.10) and (1.8), we get

$$
\begin{aligned}
T_{r}\left(B_{k+1}\right) & =n \frac{\left\|y_{k}\right\|^{2}}{y_{k}^{T} s_{k}} \\
& \leq n L^{2} \frac{\left\|s_{k}\right\|^{2}}{-(1-\beta) g_{k}^{T} s_{k}} \\
& =t_{k} \frac{n L^{2}}{1-\beta} \frac{\left\|H_{k} g_{k}\right\|^{2}}{g_{k}^{T} H_{k} g_{k}}, \\
& =c_{1} t_{k} \frac{\left\|H_{k} g_{k}\right\|^{2}}{g_{k}^{T} H_{k} g_{k}} \\
& \leq c_{1} t_{k} T_{r}\left(H_{k}\right),
\end{aligned}
$$

where $c_{1}=\frac{n L^{2}}{1-\beta}$. Combining with (3.15) yields

$$
T_{r}\left(B_{k+1}\right) \leq c_{1} t_{k}\left(\frac{n}{1-\beta}\right)^{k-1} T_{r}\left(H_{1}\right) \prod_{i=1}^{k} t_{k} .
$$

Adding above inequality to (3.15), we obtain $T_{r}\left(B_{k+1}\right)+T_{r}\left(H_{k+1}\right) \leq \frac{n}{(1-\beta)^{k}} T_{r}\left(H_{1}\right)\left[1+\frac{c_{1}(1-\beta)}{n}\right] \prod_{i=1}^{k} t_{k}$.
Now, if we denote the eigenvalues of $B_{k}$ by $h_{k 1}, h_{k 2}, \cdots, h_{k n}$, then the eigenvalues of $H_{k}$ are $\frac{1}{h_{k 1}}, \frac{1}{h_{k 2}}, \cdots, \frac{1}{h_{k n}}$, so we have

$$
T_{r}\left(B_{k+1}\right)+T_{r}\left(H_{k+1}\right)=\sum_{i=1}^{n}\left(h_{k i}+\frac{1}{h_{k i}}\right) \geq 2 n .
$$

Thus we have

$$
\prod_{i=1}^{k} t_{k} \geq \frac{2 n}{\left(\frac{n}{1-\beta}\right)^{k} T_{r}\left(H_{1}\right)\left[1+\frac{c_{1}(1-\beta)}{n}\right]} .
$$

Hence there exists a positive constant $C$ such that

$$
\prod_{i=1}^{k} t_{k} \geq c^{k}
$$

Therefore

$$
\sum_{i=1}^{k} t_{k} \geq c k
$$

Theorem 3.1 Supposed that Assumption A and B hold, and $x_{k}$ is generated by Algorithm 1.Then

$$
\liminf _{k \rightarrow \infty}\left\|g_{k}\right\|=0 \text {. }
$$

Proof. Suppose that the conclusion doesn't hold, i.e., there exists a positive constant $\varepsilon$ such that

$$
\left\|g_{k}\right\| \geq \varepsilon .
$$

From (3.3), (3.10) and Lemma 3.3, we have

$$
\begin{aligned}
T_{r}\left(B_{k+1}\right) & =n \frac{\left\|y_{k}\right\|^{2}}{y_{k}^{T} s_{k}} \\
& \leq n \frac{\left\|y_{k}\right\|^{2}}{t_{k}(1-\beta) g_{k}^{T} H_{k} g_{k}} \\
& =n \frac{\left\|y_{k}\right\|^{2}\left\|g_{k}\right\|^{2}}{t_{k}\left\|g_{k}\right\|^{2}(1-\beta) g_{k}^{T} H_{k} g_{k}} \\
& \leq \frac{n L^{2}\left\|s_{k}\right\|^{2}}{(1-\beta) \varepsilon^{2} t_{k}} \frac{\left\|g_{k}\right\|^{2}}{g_{k}^{T} H_{k} g_{k}} \\
& \leq \frac{n L^{2}\left\|s_{k}\right\|^{2}}{(1-\beta) \varepsilon^{2} t_{k}} T_{r}\left(B_{k}\right) \\
& \leq \cdots \\
& \leq\left(\frac{n L^{2}}{(1-\beta) \varepsilon^{2}}\right)^{k} T_{r}\left(B_{1}\right)\left[\left(\prod_{i=1}^{k}\left\|s_{i}\right\|^{2}\right) /\left(\prod_{i=1}^{k} t_{i}\right)\right] \\
& =c_{2}^{k} T_{r}\left(B_{1}\right) \prod_{i=1}^{k} t_{i}\left\|d_{i}\right\|^{2},
\end{aligned}
$$

where $c_{2}=\frac{n L^{2}}{(1-\beta) \varepsilon^{2}}$. From (3.4) we may obtain that 
there exists a positive constant $k_{0}$ such that

$$
t_{i}\left\|d_{i}\right\|^{2} \leq \frac{1}{c_{2}} \text { for all } i \geq k_{0} .
$$

Therefore

$$
\begin{aligned}
T_{r}\left(B_{k+1}\right) & \leq\left(c_{2}\right)^{k_{0}}\left(c_{2}\right)^{k-k_{0}} T_{r}\left(B_{1}\right) \prod_{i=1}^{k_{0}} t_{i}\left\|d_{i}\right\|^{2} \prod_{i=k_{0}+1}^{k} t_{i}\left\|d_{i}\right\|^{2} \\
& \leq\left(c_{2}\right)^{k_{0}} T_{r}\left(B_{1}\right) \prod_{i=1}^{k_{0}} t_{i}\left\|d_{i}\right\|^{2}\left(\underline{\underline{\Delta}} c_{3}\right) \\
& \leq c_{3}(k+1) \\
& \leq \frac{c_{3}}{c} \sum_{i=1}^{k+1} t_{i} .
\end{aligned}
$$

Hence from the above inequality and Lemma 3.2 and Lemma 3.3, we have

$$
\begin{aligned}
\sum_{i=1}^{\infty}\left(\frac{t_{k}}{\sum_{i=1}^{k} t_{k}}\right) & \leq \frac{c_{3}}{c} \sum_{i=1}^{\infty} \frac{t_{k}}{T_{r}\left(B_{k}\right)} \\
& \leq \frac{c_{3}}{c} \sum_{i=1}^{\infty} \frac{t_{k} g_{k}^{T} H_{k} g_{k}}{\left\|g_{k}\right\|^{2}} \\
& \leq \frac{C_{3}}{c \varepsilon^{2}} \sum_{i=1}^{\infty} t_{k} g_{k}^{T} H_{k} g_{k}<\infty .
\end{aligned}
$$

Therefore

$$
\sum_{i=1}^{\infty} t_{k}<\infty,
$$

which contradicts to Lemma 3.3. Thus (3.16) holds.

Remark: In this remark, we give a cautious update of Perry-Shanoo's Memoryless quasi-Newton method (CPSMQN), we do not discuss the global convergence here. We let it to be a topic of further research.

Algorithm 2: (CPSMQN method)

Step 0: Given $x_{1} \in R^{n}$, set $d_{1}=-g_{1}, k=1$. If $g_{1}=0$, then stop.

Step 1: Find a $t_{k}>0$ satisfying WWP.

Step 2: Let $x_{k+1}=x_{k}+t_{k} d_{k}$ and $g_{k+1}=g\left(x_{k+1}\right)$. If $g_{k+1}=0$, then stop.

Step 3: Choose $B_{k+1}$ by the following equation and Generate $d_{k+1}$,

$$
B_{k+1}=\left\{\begin{array}{cc}
\frac{\left\|y_{k}\right\|^{2}}{y_{k}^{T} s_{k}} I+\frac{y_{k} y_{k}^{T}}{y_{k}^{T} s_{k}}-\frac{\left\|y_{k}\right\|^{2}}{s_{k}^{T} y_{k}\left\|s_{k}\right\|^{2}} s_{k} s_{k}^{T} \text { if } \frac{-g_{k}^{T} s_{k}}{\left\|s_{k}\right\|^{2}} \geq m, \\
B_{k} & \text { else }
\end{array}\right.
$$

where $m$ is a positive constant.

\section{Numerical Experiments}

In this section, we report the numerical results for PSM-
QN, MPSMQN and CPSMQN method. The problems that we tested are from [4]. For each test problem, the termination condition is

$$
\left\|g\left(x_{k}\right)\right\| \leq 10^{-5} .
$$

We will test the following three methods:

-PSMQN: the Perry-Shanno's Memoryless quasiNewton method with the WWP, where $\delta=0.1$ and $\sigma=0.9$;

-MPSMQN: Algorithm 1 with $\delta=0.1, \sigma=0.9$, $\varepsilon_{1}=10^{-16}$ and $\mu=10$;

- CPSMQN: Algorithm 2 with $\delta=0.1, \sigma=0.9$ and $m=10^{-18}$.

In order to rank the iterative numerical methods, one can compute the total number of function and gradient evaluations by the formula

$$
N_{\text {total }}=N F+m * N G,
$$

where $N F, N G$ denote the number of function evaluations and gradient evaluations, respectively, and $m$ is some integer. According to the results on automatic differentiation ([5] and [6]), the value of $m$ can be set to $m=5$. That is to say, one gradient evaluation is equivalent to $m$ number of function evaluations if automatic differentiation is used.

Tables 1 shows the computation results, where the columns have the following meanings:

Problem: the name of the test problem in MATLAB;

Dim: the dimension of the problem;

NI: the number of iterations;

NF: the number of function evaluations;

NG: the number of gradient evaluations;

SD: the number of iterations for which the steepest descent direction used;

In this part, we compare the PSMQN, MPSMQN and CPSMQN method as follows: for each testing example $i$, compute the total numbers of function evaluations and gradient evaluations required by the evaluated method $j(E M(j))$ and the PSMQN method by the formula (4.1), and denote them by $N_{\text {total, },(E M(j))}$ and $N_{\text {total, }, \text { (PSMQN) }}$; then calculate the ratio

$$
r_{i}(E M(j))=\frac{N_{\text {total }, i(\operatorname{EM}(j))}}{N_{\text {total }, i(\text { PSMQN })}} .
$$

If $\operatorname{EM}\left(j_{0}\right)$ does not work for example $i_{0}$, we replace the $N_{\text {total } i_{0}\left(\operatorname{EM}\left(j_{0}\right)\right)}$ by a positive constant $\tau$ which define as follows

$$
\tau=\max \left\{N_{\text {total }, i(E M(j))}:(i, j) \notin S_{1}\right\},
$$

where

$$
S_{1}=\{(i, j) \text { : } \text { method } j \text { does not work for example } i\} \text {. }
$$


Table 1. Test results for PSMQN/MPSMQN/CPSMQN.

\begin{tabular}{|c|c|c|c|c|}
\hline & & PSMQN & MPSMQN & CPSMQN \\
\hline Problems & Dim & NI/NF/NG/SD & NI/NF/NG/SD & NI/NF/NG/SD \\
\hline ROSE & 2 & 64/133/86/0 & 65/143/82/0 & 64/133/86/0 \\
\hline FROTH & 2 & $50 / 137 / 68 / 0$ & $34 / 56 / 35 / 0$ & $50 / 137 / 68 / 0$ \\
\hline BADSCP & 2 & $161 / 558 / 324 / 3$ & $161 / 558 / 324 / 3$ & $123 / 408 / 279 / 1$ \\
\hline BADSCB & 2 & 48/255/56/4 & 22/35/23/0 & $48 / 255 / 56 / 4$ \\
\hline BEALE & 2 & $36 / 74 / 43 / 0$ & $36 / 74 / 43 / 0$ & $36 / 74 / 43 / 0$ \\
\hline JENSAM & 2 & $37 / 69 / 46 / 0$ & $37 / 69 / 46 / 0$ & $37 / 69 / 46 / 0$ \\
\hline HELIX & 3 & $53 / 77 / 57 / 0$ & $53 / 77 / 57 / 0$ & $53 / 77 / 57 / 0$ \\
\hline BARD & 3 & $92 / 117 / 95 / 0$ & $92 / 117 / 95 / 0$ & $92 / 117 / 95 / 0$ \\
\hline GAUSS & 3 & $6 / 14 / 10 / 0$ & $6 / 14 / 10 / 0$ & $6 / 14 / 10 / 0$ \\
\hline MEYER & 3 & - & - & - \\
\hline GULF & 3 & $1 / 4 / 2 / 0$ & $1 / 4 / 2 / 0$ & $1 / 4 / 2 / 0$ \\
\hline BOX & 3 & $100 / 170 / 113 / 0$ & $100 / 170 / 113 / 0$ & $100 / 170 / 113 / 0$ \\
\hline SING & 4 & $147 / 212 / 159 / 0$ & $175 / 237 / 182 / 0$ & $147 / 212 / 159 / 0$ \\
\hline WOOD & 4 & $138 / 311 / 248 / 0$ & $138 / 311 / 248 / 0$ & $139 / 321 / 244 / 1$ \\
\hline KOWOSB & 4 & $142 / 200 / 148 / 0$ & $142 / 200 / 148 / 0$ & $142 / 200 / 148 / 0$ \\
\hline BD & 4 & - & - & - \\
\hline OSB1 & 5 & - & - & - \\
\hline BIGGS & 6 & $518 / 757 / 554 / 0$ & $518 / 757 / 554 / 0$ & $518 / 757 / 554 / 0$ \\
\hline OsB2 & 11 & $610 / 717 / 620 / 0$ & $610 / 717 / 620 / 0$ & $610 / 717 / 620 / 0$ \\
\hline WATSON & 20 & - & - & - \\
\hline \multirow[t]{3}{*}{ ROSEX } & 8 & $55 / 115 / 73 / 0$ & $54 / 114 / 66 / 0$ & $55 / 115 / 73 / 0$ \\
\hline & 50 & 53/99/68/0 & 53/99/68/0 & 53/99/68/0 \\
\hline & 100 & 75/149/97/0 & 75/149/97/0 & 75/149/97/0 \\
\hline SINGX & 4 & $147 / 212 / 159 / 0$ & $147 / 212 / 159 / 0$ & $147 / 212 / 159 / 0$ \\
\hline PEN1 & 2 & 7/13/9/0 & 7/13/9/0 & 7/13/9/0 \\
\hline \multirow[t]{2}{*}{ PEN2 } & 4 & $28 / 44 / 31 / 0$ & $28 / 44 / 31 / 0$ & 28/44/31/0 \\
\hline & 50 & $481 / 845 / 539 / 0$ & $481 / 845 / 539 / 0$ & $481 / 845 / 539 / 0$ \\
\hline \multirow[t]{4}{*}{ VARDIM } & 2 & $5 / 13 / 6 / 0$ & $5 / 13 / 6 / 0$ & $5 / 13 / 6 / 0$ \\
\hline & 50 & $24 / 65 / 28 / 0$ & $24 / 65 / 28 / 0$ & $24 / 65 / 28 / 0$ \\
\hline & 100 & $30 / 77 / 33 / 0$ & $30 / 77 / 33 / 0$ & $30 / 77 / 33 / 0$ \\
\hline & 200 & $40 / 135 / 41 / 2$ & $40 / 135 / 41 / 2$ & $40 / 135 / 41 / 2$ \\
\hline \multirow[t]{3}{*}{ TRIG } & 3 & 26/31/27/0 & 26/31/27/0 & 26/31/27/0 \\
\hline & 50 & $52 / 59 / 53 / 0$ & $52 / 59 / 53 / 0$ & $52 / 59 / 53 / 0$ \\
\hline & 100 & $54 / 60 / 55 / 0$ & $54 / 60 / 55 / 0$ & $54 / 60 / 55 / 0$ \\
\hline \multirow[t]{2}{*}{$\mathrm{BV}$} & 3 & 26/40/27/0 & 26/40/27/0 & 26/40/27/0 \\
\hline & 10 & $177 / 200 / 178 / 0$ & $177 / 200 / 178 / 0$ & $177 / 200 / 178 / 0$ \\
\hline \multirow[t]{5}{*}{$\mathrm{IE}$} & 3 & $7 / 9 / 8 / 0$ & 7/9/8/0 & $7 / 9 / 8 / 0$ \\
\hline & 50 & $8 / 10 / 9 / 0$ & $8 / 10 / 9 / 0$ & $8 / 10 / 9 / 0$ \\
\hline & 100 & $8 / 10 / 9 / 0$ & $8 / 10 / 9 / 0$ & $8 / 10 / 9 / 0$ \\
\hline & 200 & $8 / 10 / 9 / 0$ & $8 / 10 / 9 / 0$ & $8 / 10 / 9 / 0$ \\
\hline & 500 & $9 / 11 / 10 / 0$ & $9 / 11 / 10 / 0$ & $9 / 11 / 10 / 0$ \\
\hline \multirow[t]{4}{*}{ TRID } & 3 & $21 / 30 / 22 / 0$ & $21 / 30 / 22 / 0$ & $21 / 30 / 22 / 0$ \\
\hline & 50 & $30 / 39 / 31 / 0$ & $30 / 39 / 31 / 0$ & $30 / 39 / 31 / 0$ \\
\hline & 100 & $32 / 39 / 33 / 0$ & $32 / 39 / 33 / 0$ & $32 / 39 / 33 / 0$ \\
\hline & 200 & $63 / 74 / 64 / 0$ & $63 / 74 / 64 / 0$ & $63 / 74 / 64 / 0$ \\
\hline BAND & 2 & $11 / 21 / 12 / 0$ & $11 / 21 / 12 / 0$ & $11 / 21 / 12 / 0$ \\
\hline \multirow[t]{4}{*}{ LIN } & 2 & $1 / 3 / 2 / 0$ & $1 / 3 / 2 / 0$ & $1 / 3 / 2 / 0$ \\
\hline & 50 & $1 / 3 / 2 / 0$ & $1 / 3 / 2 / 0$ & $1 / 3 / 2 / 0$ \\
\hline & 500 & $1 / 3 / 2 / 0$ & $1 / 3 / 2 / 0$ & $1 / 3 / 2 / 0$ \\
\hline & 1000 & $1 / 3 / 2 / 0$ & $1 / 3 / 2 / 0$ & $1 / 3 / 2 / 0$ \\
\hline \multirow[t]{2}{*}{ LIN1 } & 2 & $2 / 10 / 3 / 0$ & $2 / 10 / 3 / 0$ & $2 / 10 / 3 / 0$ \\
\hline & 10 & $2 / 21 / 3 / 0$ & $2 / 21 / 3 / 0$ & $2 / 21 / 3 / 0$ \\
\hline LIN2 & 4 & $2 / 11 / 3 / 0$ & $2 / 14 / 3 / 0$ & $2 / 14 / 3 / 0$ \\
\hline
\end{tabular}


Table 2. Relative efficiency of PSMQN, MPSMQN and CPSMQN.

\begin{tabular}{ccc}
\hline PSMQN & MPSMQN & CPSMQN \\
\hline 1 & 0.9752 & 0.9963 \\
\hline
\end{tabular}

The geometric mean of these ratios for method $\operatorname{EM}(j)$ over all the test problems is defined by

$$
r(E M(j))=\left(\prod_{i \in S} r_{i}(E M(j))\right)^{1 /|S|},
$$

where $S$ denotes the set of the test problems and $|S|$ the number of elements in $S$. One advantage of the above rule is that, the comparison is relative and hence does not be dominated by a few problems for which the method requires a great deal of function evaluations and gradient functions.

From Table 2, we observe that the average performances of the Algorithm 1 are the best among the three methods, and the average performances of the Algorithm 2 are little better than the PSMQN method. Therefore, the Algorithm 1 is the best method among the three methods given in this paper from both theory and the computational point of view.

\section{References}

[1] J. M. Perry, "A Class of Conjugate Algorithms with a Two Step Variable Metric Memory,” Discussion paper 269, Northwestern University, 1977.

[2] D. F. Shanno, "On the Convergence of a New Conjugate Gradient Algorithm,” SIAM Journal on Numerical Analysis, Vol. 15, No. 6, 1978, pp. 1247-1257. doi:10.1137/0715085

[3] J. Y. Han, G. H. Liu and H. X. Yin, "Convergence of Perry and Shanno's Memoryless Quasi-Newton Method of Nonconvex Optimization Problems," On Transactions, Vol. 1, No. 3, 1997, pp. 22-28.

[4] J. J. More, B. S. Garbow and K. E. Hillstrome, "Testing Unconstrained Optimization Software,” ACM Transactions on Mathematical Software, Vol. 7, No. 1, 1981, pp. 17-41. doi:10.1145/355934.355936

[5] A. Griewank, “On Automatic Differentiation,” Kluwer Academic Publishers, Norwell, 1989.

[6] Y. Dai and Q. Ni, “Testing Different Conjugate Gradient Methods for Large-Scale Unconstrained Optimization," Journal of Computational Mathematics, Vol. 21, No. 3, 2003, pp. 311-320. 